PROCEEDINGS OF THE

AMERICAN MATHEMATICAL SOCIETY

Volume 130, Number 5, Pages 1493-1501

S 0002-9939(01)06208-6

Article electronically published on October 23, 2001

\title{
CENTRAL LIMIT THEOREM FOR BANACH SPACE VALUED FUZZY RANDOM VARIABLES
}

\author{
FRANK N. PROSKE AND MADAN L. PURI
}

(Communicated by Claudia M. Neuhauser)

\begin{abstract}
In this paper we prove a central limit theorem for Borel measurable nonseparably valued random elements in the case of Banach space valued fuzzy random variables.
\end{abstract}

\section{INTRODUCTION}

In this paper we plan to derive a central limit theorem (CLT) for independent and identically distributed fuzzy random variables with compact $\alpha$-level sets, extending similar results for random sets (see Giné, Hahn and Zinn [6], Weil [14]). Here we are concerned with the concept of a fuzzy random variable (frv) introduced by Puri and Ralescu [12].

Fuzzy random variables are suitable for representing random mechanisms with vague outcomes of non-statistical nature. Consider for example an opinion poll where people are randomly chosen and asked about the weather. The resulting data of this random experiment arise from the imprecision of human knowledge, expressed by opinions like "warm" or "cold" which can be described by fuzzy sets rather than by a single real number or subsets of real numbers.

In section 2 we discuss some facts about frv's and other preliminaries which are needed in section 3 to present our main result, a CLT for separably Banach space valued frv's, independent and identically distributed. Klement, Puri and Ralescu [ 8$]$ proved such a result for frv's with fuzzy set values whose compact $\alpha$-level sets fulfill a Lipschitz condition with respect to the Hausdorff distance. From the practical point of view, this means a rather strong restriction of frv's to a subclass of compact fuzzy sets. Their main tools in proving the CLT are based on embedding theorems and results from probability theory on Banach spaces.

The CLT, given here, is a generalization of the latter authors' result, because no Lipschitz condition is needed. In order to then face the non-separability of involved metric spaces of fuzzy sets, our main approach is to identify linear isometrically each frv with convex and compact $\alpha$-level sets with an empirical process in $l^{\infty}(T)$ to solve a Donsker problem (see van der Vaart and Wellner [13]).

Weil [14] proved a CLT for $R^{p}$-valued compact random sets (i.e. random sets whose values are compact subsets of $R^{p}$ ). Later on these results were extended by Giné, Hahn and Zinn [6] and Puri and Ralescu [11] to the case of separable Banach

Received by the editors May 20, 2000 and, in revised form, November 22, 2000.

2000 Mathematics Subject Classification. Primary 60F05; Secondary 46B09.

(C)2001 American Mathematical Society 
spaces. Our result is a natural extension of all previous CLT's for random sets, because no Lipschitz condition is imposed.

In the sequel, we confine ourselves to complete probability spaces $(\Omega, \Im, P)$.

\section{Preliminaries}

Let $(X,\|\cdot\|)$ be a real separable Banach space.

A fuzzy set of $X$ is a function $u: X \longrightarrow[0,1]$. For each fuzzy set $u$ the $\alpha$-level set is defined by $L_{\alpha} u:=\{x \in X \mid u(x) \geq \alpha\}, 0<\alpha \leq 1$. By supp $u$ we understand the closure of $\{x \in X \mid u(x)>0\}$ in $X$. By $F_{c}(X)$, we denote the collection of those fuzzy sets $u: X \longrightarrow[0,1]$ which have the following properties: (i) $L_{\alpha} u$ is compact for all $0<\alpha \leq 1$; (ii) $\operatorname{supp} u$ is compact; (iii) $\{x \in X \mid u(x)=1\} \neq \emptyset$.

In the sequel, we set $L_{0} u=\operatorname{supp} u$. Zadeh's Extension principle [15] supplies a linear structure in $F_{c}(X)$ by the operations

$$
\begin{aligned}
(u+v)(x) & :=\sup _{y+z=x} \min (u(y), v(z)), \\
(\lambda u)(x) & := \begin{cases}u\left(\lambda^{-1} x\right), & \text { if } \lambda \neq 0, \\
\chi_{\{0\}}(x), & \text { if } \lambda=0,\end{cases} \\
\text { for } u, v & \in F_{c}(X), \lambda \in R .
\end{aligned}
$$

For later use, we define $U_{c}(X)$ as the collection of all $u \in F_{c}(X)$ with convex $\alpha$-level sets and denote the closure of the convex hull of $u \in F_{c}(X)$ by $\overline{c o} u \in F_{c}(X)$, determined by its convex compact $\alpha$-level sets $L_{\alpha} \overline{c o} u:=\overline{c o}\left(L_{\alpha} u\right), 0<\alpha \leq 1$ (see Klement, Puri and Ralescu [8]), where $\overline{c o} A$ is the closure of the convex hull of a subset $A \subseteq X$ in $X$.

Denote by $\mathcal{K}(X)(\operatorname{coK}(X))$ the class of all non-empty (convex and) compact subsets of $X$. The space $\mathcal{K}(X)(\operatorname{coK}(X))$ together with the following operations forms a linear Abelian semigroup:

$$
\begin{aligned}
& A+B:=\{a+b \mid a \in A, b \in B\}, \lambda A:=\{\lambda a \mid a \in A\} \\
& \text { for } A, B \in \mathcal{K}(X)(\operatorname{coK}(X)), \lambda \in R \text {. }
\end{aligned}
$$

Then, it is easy to check that (see Dunford and Schwartz [5], Puri and Ralescu [10, 12])

$$
L_{\alpha}(u+v)=L_{\alpha} u+L_{\alpha} v, L_{\alpha}(\lambda u)=\lambda L_{\alpha} u, \quad \lambda>0,
$$

and

$$
\overline{c o}\left(L_{\alpha} u+L_{\alpha} v\right)=\overline{c o} L_{\alpha} u+\overline{c o} L_{\alpha} v, \overline{c o}\left(\lambda L_{\alpha} u\right)=\lambda \overline{c o} L_{\alpha} u, \quad \lambda>0,
$$

for all $u, v \in F_{c}(X), 0<\alpha \leq 1$. Also $F_{c}(X)$ and $U_{c}(X)$ are closed under the above operations and form like $\mathcal{K}(X)$ or $\operatorname{co} \mathcal{K}(X)$ a linear Abelian semigroup.

$\mathcal{K}(X)$ is metrizable by the well-known Hausdorff distance $d_{H}$, given by

$$
d_{H}: \mathcal{K}(X) \times \mathcal{K}(X) \longrightarrow[0, \infty) ;(A, B) \mapsto \max \left\{\sup _{a \in A} \inf _{b \in B}\|a-b\|, \sup _{b \in B} \inf _{a \in A}\|a-b\|\right\}
$$

$\left(\mathcal{K}(X), d_{H}\right)$ and $\left(\operatorname{co} \mathcal{K}(X), d_{H}\right)$ are then complete separable metric spaces (see Puri and Ralescu [11]). By identifying each $A \in \mathcal{K}(X)$ with its characteristic function $\chi_{A} \in F_{c}(X)$, we can generalize the Hausdorff distance to the case of $F_{c}(X)$, getting a continuous semigroup monomorphism: 
Proposition 1 (see Puri and Ralescu 12]). $\left(F_{c}(X), d_{\infty}\right)\left(\left(U_{c}(X), d_{\infty}\right)\right)$ is a complete, but in contrast to $\left(\mathcal{K}(X), d_{H}\right)\left(\left(\operatorname{coK}(X), d_{H}\right)\right)$, a nonseparable metric space, endowed with the metric $d_{\infty}$ defined by $d_{\infty}(u, v)=\sup _{0<a<1} d_{H}\left(L_{\alpha} u, L_{\alpha} v\right)$ for $u, v \in F_{c}(X)$.

Let $(\Omega, \Im, P)$ be a probability space. A fuzzy random variable (frv) is a Borel measurable function $\xi: \Omega \longrightarrow\left(F_{c}(X), d_{\infty}\right)$. As one can see, frv's generalize compact random sets $A$ (i.e. Borel measurable functions $A: \Omega \longrightarrow\left(\mathcal{K}(X), d_{H}\right)$ ) by identification with the characteristic function $\chi_{A}$.

Proposition 2 (see Klement, Puri and Ralescu [8]). Let $\xi: \Omega \longrightarrow\left(F_{c}(X), d_{\infty}\right)$ be an frv. Then:

(i) $\overline{c o} \xi: \Omega \longrightarrow\left(U_{c}(X), d_{\infty}\right) ; \omega \mapsto \overline{c o} \xi(\omega)$ is an frv.

(ii) $L_{\alpha} \xi: \Omega \longrightarrow\left(\mathcal{K}(X), d_{H}\right) ; \omega \mapsto L_{\alpha} \xi(\omega)$ and

$\overline{c o}\left(L_{\alpha} \xi\right): \Omega \longrightarrow\left(\operatorname{coK}(X), d_{H}\right) ; \omega \longmapsto \overline{c o}\left(L_{\alpha} \xi(\omega)\right)$ are (convex) compact random sets for all $0<\alpha \leq 1$.

(iii) supp $\xi: \Omega \longrightarrow\left(\mathcal{K}(X), d_{H}\right) ; \omega \mapsto L_{0} \xi(\omega)$ is a compact random set.

Proposition 3 (see Puri and Ralescu [12]). Let $\xi: \Omega \longrightarrow\left(F_{c}(X), d_{\infty}\right)$ be an frv with $E\|\xi\|_{\infty}<\infty\left(\|\xi(\omega)\|_{\infty}:=\sup _{\alpha>0} d_{H}\left(\{0\}, L_{\alpha} \xi(\omega)\right)\right)$. Then there exists a unique fuzzy set $u \in U_{c}(X)$ such that the $\alpha$-level sets of $u$ are the Aumann expectations of the convex compact random sets $\overline{c o}\left(L_{\alpha} \xi\right)$ (see for definition Aubin, Frankowska [2], Debreu [4]), i.e. $L_{\alpha} u=E \overline{c o}\left(L_{\alpha} \xi\right)$ for all $0<\alpha \leq 1$.

If $\xi: \Omega \longrightarrow\left(F_{c}(X), d_{\infty}\right)$ is an frv with $E\|\xi\|_{\infty}<\infty$, then, by the last result, the expected value $E \xi$ is the unique convex fuzzy set satisfying the property $L_{\alpha} E \xi=$ $E \overline{c o}\left(L_{\alpha} \xi\right), 0<\alpha \leq 1$.

One of our main tools in proving the CLT is a result for embedding convex compact sets into the space of continuous functions which stems in this form from Giné, Hahn and Zinn [6], but is originally due to Hörmander [7]:

Theorem 4. The function $j:\left(\operatorname{coK}(X), d_{H}\right) \longrightarrow\left(C\left(\right.\right.$ ball $\left.\left.X^{*}, d_{*}\right),\|\cdot\|_{\infty}\right)\left(\|\cdot\|_{\infty}\right.$ is the supremum norm $)$, given by $A \longmapsto\left(f \mapsto \sup _{a \in A} f(a)\right)$, satisfies:

(i) $j$ is an isometry (i.e. $\left.d_{H}(A, B)=\|j(A)-j(B)\|_{\infty}\right)$.

(ii) $j(A+B)=j(A)+j(B), j(\lambda A)=\lambda j(A)(\lambda \geq 0)$.

$d_{*}$ denotes a metric inducing the weak* topology of ball $X^{*}$, where ball $X^{*}$ is the norm-closed unit ball of the dual of $X$.

A crucial idea in deriving a CLT for $F_{c}\left(R^{p}\right)$-valued frv's (see Corollary 6.1) is to first reduce the study of frv's to the convex case by convexifying the underlying variables and then to get rid of the convexity restriction by applying the following inequality:

Theorem 5 (Shapley-Folkman-Starr). For all $A_{1}, \ldots, A_{n} \in \mathcal{K}\left(R^{p}\right)$ and $n \in \mathbb{N}$

$$
d_{H}\left(A_{1}+\ldots+A_{n}, \overline{c o}\left(A_{1}+\ldots+A_{n}\right)\right) \leq \sqrt{p} \max _{1 \leq i \leq n}\left\|A_{i}\right\|\left(\|A\|:=d_{H}(\{0\}, A)\right) .
$$

We wish to employ the theory of empirical processes (see van der Vaart and Wellner [13]) to investigate frv's. To this end, we need the term of a bracketing number to measure the "size" of certain function classes: Let $(\mathcal{F},\|\cdot\|)$ be a subset of a normed space of real functions $f: \chi \longrightarrow R$ on a set $\chi$. Then the bracket $[l, u]$ for real functions $l, u$ is the set of all functions $f: \chi \longrightarrow R$ satisfying $l \leq f \leq u$. 
An $\varepsilon$-bracket is a bracket $[l, u]$ with $\|l-u\|<\varepsilon$. Then the bracketing number $N_{[]}(\varepsilon, \mathcal{F},\|\cdot\|)$ is the minimal number of $\varepsilon$-brackets covering $\mathcal{F}$. Note that in the definition of the bracketing number, the functions $l$ and $u$ are assumed to have finite norms, but not to belong to $\mathcal{F}$ itself.

\section{Central limit theorem for fuzzy RAndom variables}

In order to prepare for our main result, let us state two more definitions: If $(T, d)$ is a metric space, the $\varepsilon$-entropy of $T$, denoted by $N(\varepsilon, T, d)(\varepsilon>0)$, is the minimal number of balls of radius $<\varepsilon$, with centres in $T$ which cover $T$. The Minkowski functional $\|\cdot\|_{K}$ of a convex, compact, symmetric subset $K$ of $X$ is given by $\|A\|_{K}=\inf \{\lambda>0 \mid A \subseteq \lambda K\}(\inf \emptyset:=\infty)$.

Theorem 6 (CLT for frv's). Let $(X,\|\cdot\|)$ be a separable Banach space over $R$, and $\xi_{i}: \Omega \longrightarrow\left(U_{c}(X), d_{\infty}\right)$ an i.i.d. sequence of frv's such that:

(i) there exists a convex, compact, symmetric $K \subseteq X$ such that $0<E \|$ supp $\xi_{1} \|_{K}^{2}$ $<\infty$ and $\int_{0}^{1}\left(\log N\left(\varepsilon, \text { ball } X^{*}, d_{K}\right)\right)^{1 / 2} \lambda^{1}(d \varepsilon)<\infty$, where $d_{K}$ is the semimetric on ball $X^{*}$ with $d_{K}\left(f_{1}, f_{2}\right)=\sup _{a \in K}\left|f_{1}(a)-f_{2}(a)\right|$, and

(ii) $\int_{0}^{1}\left(\log N\left(\varepsilon^{2}, K,\|\cdot\|\right)\right)^{1 / 2} \lambda^{1}(d \varepsilon)<\infty$.

Then, a CLT in the following sense is valid:

$$
\sqrt{n} d_{\infty}\left(\sum_{i=1}^{n} \xi_{i} / n, E \xi_{1}\right) \longrightarrow\|\underline{Y}\|_{\infty} \text { weakly }
$$

for a tight Gaussian process $\underline{Y}$ in $l^{\infty}\left([0,1] \times\right.$ ball $\left.X^{*}\right)$.

Proof. For proving this result we aim at applying a CLT for empirical processes (see Theorem 2.11.23, p. 221 in van der Vaart and Wellner [13]), which comes down to bounding above bracketing numbers in an appropriate way for the function class $\mathcal{F}:=\left\{f_{t} \mid t \in T\right\}$, consisting of $\mathcal{B}\left(U_{c}(X)\right), \mathcal{B}(R)$-measurable functions $f_{t}$ : $\left(U_{c}(X), d_{\infty}\right) \longrightarrow R ; u \longmapsto \sup _{a \in L_{\alpha} u} f(a)$ for $t=(\alpha, f) \in T:=[0,1] \times$ ball $X^{*}$. In the sequel we adopt the notions in van der Vaart and Wellner [13] and define $F=\|\cdot\|_{\infty}$. The proof is accomplished step-by-step as follows:

1. The existence of the second moment of $\left\|\operatorname{supp} \xi_{1}\right\|_{K}$ implies the existence of $\left\|\xi_{1}\right\|_{\infty}$. Then, one can easily prove that there exists a unique fuzzy set $E\left(\left\|\xi_{1}\right\|_{\infty} \xi_{1}\right) \in$ $U_{c}(X)$ such that $L_{\alpha} E\left(\left\|\xi_{1}\right\|_{\infty} \xi_{1}\right)=E\left(\left\|\xi_{1}\right\|_{\infty} L_{\alpha} \xi_{1}\right)$ for all $0<\alpha \leq 1$. Let us equip the interval $[0,1]$ with the pseudodistance

$$
\rho_{1}:[0,1] \times[0,1] \longrightarrow[0, \infty) ;\left(\alpha_{1}, \alpha_{2}\right) \mapsto d_{H}^{1 / 2}\left(L_{\alpha_{1}} E\left(\left\|\xi_{1}\right\|_{\infty} \xi_{1}\right), L_{\alpha_{2}} E\left(\left\|\xi_{1}\right\|_{\infty} \xi_{1}\right)\right) .
$$

Taking into account that the $\alpha$-level sets of a fuzzy set $u \in F_{c}(X)$ are nonincreasing and that the mapping $\left(\alpha \mapsto L_{\alpha} u\right)$ on the interval $[0,1]$ is left continuous in $(0,1]$ as well as right continuous at 0 with respect to the Hausdorff distance (see Klement, Puri and Ralescu [8]), one sees that the $\rho_{1}$-balls with radius $<\varepsilon$ and centres $0 \leq \alpha_{0} \leq 1, U_{\varepsilon}^{\left(\rho_{1}\right)}\left(\alpha_{0}\right)$ are non-degenerated subintervals of $[0,1]$.

2. For convenience, we denote $f_{t}(\cdot)=f((\alpha, \widetilde{f}), \cdot)$ for $t=(\alpha, \widetilde{f})$. Using the definition of the Minkowski functional $\|\cdot\|_{K}$, we find that

$$
\left|f\left(\left(\alpha_{2}, f_{1}\right), \xi_{1}\right)-f\left(\left(\alpha_{2}, f_{2}\right), \xi_{1}\right)\right| \leq\left\|\operatorname{supp} \xi_{1}\right\|_{K} d_{K}\left(f_{1}, f_{2}\right)
$$

holds for all $\left(\alpha_{2}, f_{1}\right),\left(\alpha_{2}, f_{2}\right) \in T$. 
This leads us to following inequality:

$$
\begin{aligned}
A & :=\left|f\left(\left(\alpha_{1}, f_{1}\right), \xi_{1}\right)-f\left(\left(\alpha_{2}, f_{2}\right), \xi_{1}\right)\right|^{2} \\
& \leq 2\left(\left|f\left(\left(\alpha_{1}, f_{1}\right), \xi_{1}\right)-f\left(\left(\alpha_{2}, f_{1}\right), \xi_{1}\right)\right|^{2}+\left\|\operatorname{supp} \xi_{1}\right\|_{K}^{2} d_{K}^{2}\left(f_{1}, f_{2}\right)\right)
\end{aligned}
$$

for $\left(\alpha_{1}, f_{1}\right),\left(\alpha_{2}, f_{2}\right) \in T$.

We proceed to bound the above and obtain

$$
\begin{aligned}
\|A\|_{\mathcal{P}, 2}= & \left(E\left|f\left(\left(\alpha_{1}, f_{1}\right), \xi_{1}\right)-f\left(\left(\alpha_{2}, f_{2}\right), \xi_{1}\right)\right|^{2}\right)^{1 / 2} \\
\leq & \sqrt{2}\left(\sup _{\widetilde{f} \in \text { ball } X^{*}} E\left|f\left(\left(\alpha_{1}, \widetilde{f}\right), \xi_{1}\right)-f\left(\left(\alpha_{2}, \widetilde{f}\right), \xi_{1}\right)\right|^{2}\right)^{1 / 2} \\
& \left.+\left(E\left\|\operatorname{supp} \xi_{1}\right\|_{K}^{2}\right)^{1 / 2} d_{K}\left(f_{1}, f_{2}\right)\right) \\
\leq & K\left(\sup _{\widetilde{f} \in \text { ball } X^{*}} E\left(\left\|\xi_{1}\right\|_{\infty}\left|f\left(\left(\alpha_{1}, \widetilde{f}\right), \xi_{1}\right)-f\left(\left(\alpha_{2}, \widetilde{f}\right), \xi_{1}\right)\right|\right)\right)^{1 / 2} \\
& \left.+d_{K}\left(f_{1}, f_{2}\right)\right) \\
= & K\left(\left(\sup _{\tilde{f} \in \text { ball } X^{*}} E\left|f\left(\left(\alpha_{1}, \widetilde{f}\right),\left\|\xi_{1}\right\|_{\infty} \xi_{1}\right)-f\left(\left(\alpha_{2}, \widetilde{f}\right),\left\|\xi_{1}\right\|_{\infty} \xi_{1}\right)\right|\right)^{1 / 2}\right. \\
& \left.+d_{K}\left(f_{1}, f_{2}\right)\right) \\
= & K \rho\left(\left(\alpha_{1}, f_{1}\right),\left(\alpha_{2}, f_{2}\right)\right) \text { for all }\left(\alpha_{1}, f_{1}\right),\left(\alpha_{2}, f_{2}\right) \in T
\end{aligned}
$$

with the pseudodistance $\rho=\rho_{1}+\rho_{2}$ on $T$, where $\rho_{2}=d_{K}$.

The last relation follows from Theorem 4 and the fact that $E \sup _{a \in A} f(a)=$ $\sup _{a \in E A} f(a)\left(f \in X^{*}\right)$ is valid for the Aumann expectation of compact random sets $A$ (see Aubin and Frankowska 2]).

3. We intend to construct a finite $\delta$-net for the pseudodistance $\rho_{1}$.

For that purpose, let us define:

$\alpha_{0}:=1$.

$\alpha_{1}:=\inf \left\{\alpha \in[0,1] \mid \alpha \leq \alpha_{0}, \rho_{1}\left(\alpha, \alpha_{0}\right)<\delta\right\}$.

If $\alpha_{1}=0$, cut short the procedure, otherwise define:

$\alpha_{2}:=\inf \left\{\alpha \in[0,1] \mid \alpha \leq \alpha_{1}, \rho_{1}\left(\alpha, \alpha_{1}\right)<\delta\right\}$

.

continue by induction.

Suppose now that the procedure doesn't stop.

The left-continuity of $\left(\alpha \mapsto \rho_{1}\left(\alpha, \alpha_{n-1}\right)\right)$ on $(0,1]$ for all $n$ implies $\rho_{1}\left(\alpha_{n}, \alpha_{n-1}\right) \geq$ $\delta$ for all $n$. Because of the compactness of $L_{\alpha_{n}} E\left(\left\|\xi_{1}\right\|_{\infty} \xi_{1}\right)$ and the continuity of $\left(x \mapsto \inf _{y \in L_{\alpha_{n-1}} E\left(\left\|\xi_{1}\right\|_{\infty} \xi_{1}\right)}\|x-y\|\right)$ for all $n$, there exists a sequence $\left(a_{n}\right)_{n \in \mathbb{N}}$ in $\operatorname{supp} E\left(\left\|\xi_{1}\right\|_{\infty} \xi_{1}\right)$ such that $\left\|a_{i}-a_{j}\right\| \geq \delta^{2}$ for $i \neq j$. But this is inconsistent with the compactness of $\operatorname{supp} E\left(\left\|\xi_{1}\right\|_{\infty} \xi_{1}\right)$.

To summarize, there exists a natural number $n$ such that $\alpha_{0}=1, \alpha_{n}=0$, $\alpha_{m}<\alpha_{m-1}$ and $\rho_{1}\left(\alpha, \alpha_{m-1}\right)<\delta$ on $\left(\alpha_{m}, \alpha_{m-1}\right]$ for all $m=1, \ldots, n$. Thus $\left(\alpha_{m}\right)_{m=0}^{n}$ is a $\delta$-net for $\rho_{1}$. Since the identity map $i d:\left(\right.$ ball $\left.X^{*}, d_{*}\right) \longrightarrow\left(\right.$ ball $\left.X^{*}, d_{K}\right)$ is continuous, we conclude in addition that there is a finite $\delta$-net for $\rho$.

4. From step 3 we know that there exists a finite $\rho$ - $\delta$-net $\left(S_{i}^{(\delta)}\right)_{i=1}^{n}$. Since $U_{\delta}^{(\rho)}\left(S_{i}^{(\delta)}\right)$ is a subset of $U_{\delta}^{\left(\rho_{1}\right)}\left(\alpha_{i}{ }^{(\delta)}\right) \times U_{\delta}^{\left(\rho_{2}\right)}\left(f_{i}^{(\delta)}\right)$ for $S_{i}^{(\delta)}=\left(\alpha_{i}^{(\delta)}, f_{i}^{(\delta)}\right), i=1, \ldots, n$, 
we get

$$
\begin{aligned}
C:= & \sup _{\substack{(\alpha, \widetilde{f}) \in \\
U_{\delta}^{(\rho)}\left(S_{i}^{(\delta)}\right)}}\left|f\left((\alpha, \widetilde{f}), \xi_{1}\right)-f\left(\left(\alpha_{i}^{(\delta)}, f_{i}^{(\delta)}\right), \xi_{1}\right)\right|^{2} \\
\leq & 2(\underbrace{\sup _{\alpha \in U_{\delta}^{\left(\rho_{1}\right)}\left(\alpha_{i}^{(\delta)}\right)}\left|f\left(\left(\alpha, f_{i}^{(\delta)}\right), \xi_{1}\right)-f\left(\left(\alpha_{i}^{(\delta)}, f_{i}^{(\delta)}\right), \xi_{1}\right)\right|^{2}}_{:=f(\delta)} \\
& \left.+\left\|\operatorname{supp} \xi_{1}\right\|_{K}^{2} \sup _{\tilde{f} \in U_{\delta}^{\left(\rho_{2}\right)}\left(f_{i}^{(\delta)}\right)} d_{K}^{2}\left(\widetilde{f}, f_{i}^{(\delta)}\right)\right) .
\end{aligned}
$$

Because of step 1 and monotonicity reasons there exist sequences $\underline{\alpha}_{n} \searrow$ and $\bar{\alpha}_{n} \nearrow$ independent of $\omega \in \Omega$ and $U_{\delta}^{\left(\rho_{1}\right)}\left(\alpha_{i}^{(\delta)}\right)$ such that

$$
f(\delta)=\max \left\{\lim _{n} C_{1}^{(n)}, \lim _{n} C_{2}^{(n)}\right\}
$$

where $C_{1}^{(n)}=\left|f\left(\left(\underline{\alpha}_{n}, f_{i}^{(\delta)}\right), \xi_{1}\right)-f\left(\left(\alpha_{i}^{(\delta)}, f_{i}^{(\delta)}\right), \xi_{1}\right)\right|^{2}$, and

$$
C_{2}^{(n)}=\left|f\left(\left(\bar{\alpha}_{n}, f_{i}^{(\delta)}\right), \xi_{1}\right)-f\left(\left(\alpha_{i}^{(\delta)}, f_{i}^{(\delta)}\right), \xi_{1}\right)\right|^{2}
$$

Using the monotone convergence theorem and step 2, we find

$$
\begin{aligned}
E f(\delta) & =\lim _{n} E \max \left\{C_{1}^{(n)}, C_{2}^{(n)}\right\} \\
& \leq \lim _{n}\left(E C_{1}^{(n)}+E C_{2}^{(n)}\right) \\
& \leq \lim _{n} K\left(\rho_{1}^{2}\left(\underline{\alpha}_{n}, \alpha_{i}^{(\delta)}\right)+\rho_{1}^{2}\left(\bar{\alpha}_{n}, \alpha_{i}^{(\delta)}\right)\right) \\
& \leq \widetilde{K} \delta^{2} .
\end{aligned}
$$

Since the continuity of the expressions $\inf _{t \in A} f_{t}(\cdot)$ and $\sup _{t \in A} f_{t}(\cdot)$ for $A \subseteq T$ ensures the measurability of $C$, we can write

$$
(E C)^{1 / 2} \leq \sqrt{2}\left((E f(\delta))^{1 / 2}+\left(E\left\|\operatorname{supp} \xi_{1}\right\|_{K}^{2}\right)^{1 / 2} \delta\right) \leq K_{0} \delta .
$$

5. First, we want to find a finite number of $\varepsilon$-brackets which cover the function class $\mathcal{F}=\left\{f_{t} \mid t \in T\right\}$. In doing so, let us define for $t_{0} \in T$ and $\delta>0 f_{\delta, t_{0}}^{u}:=$ $\sup _{t \in U_{\delta}^{(\rho)}\left(t_{0}\right)} f_{t}(\cdot)$ and $f_{\delta, t_{0}}^{l}:=\inf _{t \in U_{\delta}^{(\rho)}\left(t_{0}\right)} f_{t}(\cdot)$. For the reasons mentioned at the end of step $4, f_{\delta, t_{0}}^{u}$ and $f_{\delta, t_{0}}^{l}$ are measurable functions. Because of step 3 there exists a finite $\rho$ - $\delta$-net, $S(\delta) \subseteq T$. We then see that for all $t \in T$ there is a $s \in S(\delta)$ such that for all $v \in U_{c}(X), f_{\delta, s}^{l}(v) \leq f_{t}(v) \leq f_{\delta, s}^{u}(v)$ holds.

Furthermore, using the last inequality of step 4 , we note that

$$
\begin{aligned}
\left\|f_{\delta, s}^{u}-f_{\delta, s}^{l}\right\|_{\mathcal{P}, 2} & =\left(E\left(f_{\delta, s}^{u}\left(\xi_{1}\right)-f_{\delta, s}^{l}\left(\xi_{1}\right)\right)^{2}\right)^{1 / 2} \\
& =\left(E\left(\sup _{t_{1}, t_{2} \in U_{\delta}^{(\rho)}(s)}\left|f_{t_{1}}\left(\xi_{1}\right)-f_{t_{2}}\left(\xi_{1}\right)\right|^{2}\right)\right)^{1 / 2} \\
& \leq \sqrt{2}\left(E\left(\sup _{t \in U_{\delta}^{(\rho)}(s)}\left|f_{t}\left(\xi_{1}\right)-f_{s}\left(\xi_{1}\right)\right|^{2}\right)\right)^{1 / 2} \\
& \leq K \delta \text { for all } s \in S(\delta) \text { and a constant } K .
\end{aligned}
$$


This enables us to give an upper bound for bracketing numbers by means of entropy numbers:

$$
N_{[]}\left(\varepsilon, \mathcal{F}, L_{2}(\mathcal{P})\right) \leq N(\varepsilon / K, T, \rho) .
$$

Without loss of generality we set $K=1$ and then

$$
\begin{aligned}
& \int_{0}^{1}\left(\log N_{[]}\left(\varepsilon, \mathcal{F}, L_{2}(\mathcal{P})\right)\right)^{1 / 2} \lambda^{1}(d \varepsilon) \\
\leq & \int_{0}^{1}(\log N(\varepsilon, T, \rho))^{1 / 2} \lambda^{1}(d \varepsilon) \\
\leq & \int_{0}^{1}\left(\log N\left(\varepsilon / 2,[0,1], \rho_{1}\right)\right)^{1 / 2} \lambda^{1}(d \varepsilon) \\
& +\int_{0}^{1}\left(\log N\left(\varepsilon / 2, \text { ball } X^{*}, d_{K}\right)\right)^{1 / 2} \lambda^{1}(d \varepsilon) .
\end{aligned}
$$

6. Let $\left(\alpha_{i}\right)_{i=1}^{n}$ be a finite $\delta$-net for $\rho_{1}$ with the corresponding

$$
\left(a_{i}\right)_{i=1}^{n} \subseteq \operatorname{supp} E\left(\left\|\xi_{1}\right\|_{\infty} \xi_{1}\right) \subseteq\left(E\left\|\xi_{1}\right\|_{\infty}\left\|\operatorname{supp} \xi_{1}\right\|_{K}\right) K
$$

constructed as in step 3.

We claim that $n-1 \leq N\left(\delta^{2} / 3,\left(E\left\|\xi_{1}\right\|_{\infty}\left\|\operatorname{supp} \xi_{1}\right\|_{K}\right) K,\|\cdot\|\right)=: m$.

Let us say $n-1>m$. Take $\left\{U_{\delta^{2} / 2}\left(x_{i}\right) \mid i=1, \ldots, m\right\}$, a minimal $\delta^{2} / 3$-covering of $\left(E\left\|\xi_{1}\right\|_{\infty}\left\|\operatorname{supp} \xi_{1}\right\|_{K}\right) K$ with respect to $\|\cdot\|$. We can then find pairwise disjoint sets $I_{1}, \ldots, I_{m}$ such that $\bigcup_{i=1}^{m} U_{\delta^{2} / 3}\left(x_{i}\right)=\bigcup_{i=1}^{m} I_{i}$ and $\operatorname{diam} I_{i} \leq 2 / 3 \delta^{2}$ for all $i$. Then there must be natural numbers $i_{0}, i \neq j$, with $i, j \in\{1, \ldots, n-1\}$ and $i_{0} \in\{1, \ldots, m\}$ such that $a_{i}, a_{j} \in I_{i_{0}}$. On the other hand, by construction, $\left\|a_{i}-a_{j}\right\| \geq \delta^{2}$ is valid for all $i \neq j$. But this leads to a contradiction.

Hence

$$
\begin{aligned}
& \int_{0}^{1}\left(\log N\left(\varepsilon,[0,1], \rho_{1}\right)\right)^{1 / 2} \lambda^{1}(d \varepsilon) \\
\leq & \int_{0}^{1}\left(\log 2 N\left(\varepsilon^{2} / 3,\left(E\left\|\xi_{1}\right\|_{\infty}\left\|\operatorname{supp} \xi_{1}\right\|_{K}\right) K,\|\cdot\|\right)\right)^{1 / 2} \lambda^{1}(d \varepsilon) \\
= & \int_{0}^{1}\left(\log 2 N\left(\varepsilon^{2} / 3 E\left(\left\|\xi_{1}\right\|_{\infty}\left\|\operatorname{supp} \xi_{1}\right\|_{K}\right), K,\|\cdot\|\right)\right)^{1 / 2} \lambda^{1}(d \varepsilon) .
\end{aligned}
$$

7. Finally, by the last two steps, we prove the following integral condition:

$$
\int_{0}^{1}\left(\log N_{[]}\left(\varepsilon, \mathcal{F}, L_{2}(\mathcal{P})\right)\right)^{1 / 2} \lambda^{1}(d \varepsilon)<\infty
$$

Further, we see that

$$
\begin{aligned}
\mathcal{P}^{*} F^{2} & =E\left\|\xi_{1}\right\|_{\infty}^{2}=O(1) \text { as well as } \\
\mathcal{P}^{*} F^{2} 1_{\{F>\eta \sqrt{n}\}} & =\mathcal{P}^{*} F^{2} 1_{\left\{\left\|\xi_{1}\right\|_{\infty}>\eta \sqrt{n}\right\}} \longrightarrow 0 \text { for all } \eta>0 .
\end{aligned}
$$

In addition, the last inequality of step 3 provides

$$
\begin{aligned}
\sup _{\rho(s, t)<\delta_{n}} \mathcal{P}\left(f_{s}-f_{t}\right)^{2} & =\sup _{\rho(s, t)<\delta_{n}} E\left|f_{s}\left(\xi_{1}\right)-f_{t}\left(\xi_{1}\right)\right|^{2} \\
& \leq \sup _{\rho(s, t)<\delta_{n}} K \rho^{2}(s, t) \\
& \leq K \delta_{n}^{2} \longrightarrow 0 \text { for all } \delta_{n} \downarrow 0 .
\end{aligned}
$$


With these verifications we are now ready to apply a CLT result for empirical processes (see Theorem 2.11.23, p. 221 in van der Vaart and Wellner [13]). Together with Theorem 4 and the continuous mapping theorem we obtain the desired result:

$$
\begin{aligned}
& \sqrt{n} d_{\infty}\left(\sum_{i=1}^{n} \xi_{i} / n, E \xi_{1}\right) \\
= & \sup _{t \in T}\left|n^{-1 / 2}\left(\sum_{i=1}^{n} E f_{t}\left(\xi_{1}\right)-f_{t}\left(\xi_{i}\right)\right)\right| \longrightarrow\|\underline{Y}\|_{\infty} \text { weakly } \\
& \text { for a tight Gaussian process } \underline{Y} \text { in } l^{\infty}\left([0,1] \times \text { ball } X^{*}\right) .
\end{aligned}
$$

The finite dimensional case then yields:

Corollary 6.1. If $\xi_{i}: \Omega \longrightarrow\left(F_{c}\left(R^{p}\right), d_{\infty}\right)$ is an i.i.d. sequence of frv's with $E\left\|\xi_{1}\right\|_{\infty}^{2}<\infty$, then

$$
\sqrt{n} d_{\infty}\left(\sum_{i=1}^{n} \xi_{i} / n, E \xi_{1}\right) \longrightarrow\|\underline{Y}\|_{\infty}
$$

weakly for a tight Gaussian process $\underline{Y}$ in $l^{\infty}\left([0,1] \times\right.$ ball $\left.R^{p}\right)$.

Proof. Let us first consider the i.i.d. frv's $\overline{c o} \xi_{i}: \Omega \longrightarrow\left(U_{c}\left(R^{p}\right), d_{\infty}\right)$. We choose $K=$ ball $R^{p}$. Since $\operatorname{supp} \overline{c o} \xi_{1} \subseteq\left\|\xi_{1}\right\|_{\infty}$ ball $R^{p}$ we see that $E\left\|\operatorname{supp} \overline{c o} \xi_{1}\right\|_{K}^{2}<$ $\infty$. The spaces (ball $R^{p},\|\cdot\|$ ) and (ball $\left.\left(R^{p}\right)^{*}, d_{K}\right)$ are isometrical to each other. Therefore we get $N\left(\delta\right.$, ball $\left.R^{p},\|\cdot\|\right)=N\left(\delta\right.$, ball $\left.\left(R^{p}\right)^{*}, d_{K}\right)$ for all $\delta>0$. Taking into consideration that

$$
\int_{0}^{1}\left(\log N\left(\varepsilon^{2}, K,\|\cdot\|\right)\right)^{1 / 2} \lambda^{1}(d \varepsilon) \leq \int_{0}^{1} \sqrt{p}\left(\log \left(4 \sqrt{p} / \varepsilon^{2}\right)\right)^{1 / 2} \lambda^{1}(d \varepsilon)<\infty,
$$

we follow the existence of the integrals in Theorem 6 . Thus it implies that

$$
\sqrt{n} d_{\infty}\left(\sum_{i=1}^{n} \overline{c o} \xi_{i} / n, E \xi_{1}\right) \longrightarrow\|\underline{Y}\|_{\infty}
$$

weakly for a tight Gaussian process $\underline{Y}$ in $l^{\infty}\left([0,1] \times\right.$ ball $\left.R^{p}\right)$.

Further, the Shapley-Folkman-Starr theorem gives

$$
\sqrt{n} d_{\infty}\left(\sum_{i=1}^{n} \xi_{i} / n, \sum_{i=1}^{n} \overline{c o} \xi_{i} / n\right) \leq \sqrt{p} \max _{1 \leq i \leq n}\left\|\xi_{i}\right\|_{\infty} / \sqrt{n} .
$$

We can then write

$$
\begin{aligned}
& \sqrt{n} d_{\infty}\left(\sum_{i=1}^{n} \xi_{i} / n, E \xi_{1}\right) \\
& \quad \leq \sqrt{p} \max _{1 \leq i \leq n}\left\|\xi_{i}\right\|_{\infty} / \sqrt{n}+\sqrt{n} d_{\infty}\left(\sum_{i=1}^{n} \overline{c o} \xi_{i} / n, E \xi_{1}\right) .
\end{aligned}
$$

The first term of the above inequality converges weakly to zero (see, for example, p. 188 in Breiman [3]), whereas the second converges to $\|\underline{Y}\|_{\infty}$, concluding the proof. 


\section{Conclusion}

The CLT for $U_{c}(X)$-valued frv's (Theorem 6 ) is analogous to that of Giné, Hahn and Zinn [6] for random convex compact sets in separable Banach spaces. But comparatively Theorem 6 requires in addition another integral condition. It would be worth checking out possible relations between these two integrals. Regarding the finite dimensional case we attained a natural generalization of the result of Weil [14] for random compact sets.

\section{REFERENCES}

[1] Z. Artstein and R.A. Vitale, A strong law of large numbers for random compact sets, Ann. Prob. 3 (1975), 879-882. MR 52:6825

[2] J.P. Aubin and H. Frankowska, Set-valued Analysis. Systems and Control: Foundations and Applications, Birkhäuser, 1990. MR 91d:49001

[3] L. Breiman, Probability. Addison-Wesley, 1968. MR 37:4841

[4] G. Debreu, Integration of correspondences, Proc. Fifth Berkeley Symp. Math Statist. Prob. 2 (1966), 351-372, University of California Press. MR 37:3835

[5] N. Dunford and J.T. Schwartz, Linear Operators, Part I. Interscience, 1958. MR 22:8302

[6] E. Giné, M.G. Hahn and J. Zinn, Limit theorems for random sets, Springer Lecture Notes in Math. 990 (1983), 112-135. MR 85d:60019

[7] L. Hörmander, Sur la fonction d'appui des ensembles convexe dans un espace localement convexe, Ark. Mat. 3 (1954), 181-186. MR 16:831e

[8] E.P. Klement, M.L. Puri and D.A. Ralescu, Limit theorems for fuzzy random variables, Proc. Royal Soc. Lond. A 407 (1986), 171-182. MR 88b:60092

[9] F.N. Proske, Limit theorems for fuzzy random variables, Ph.D. Thesis, Ulm University, Germany, 1997.

[10] M.L. Puri and D.A. Ralescu, Differentials of fuzzy functions, J. Math. Anal. Appl. 91 (1983), 552-558. MR 85c:26014

[11] M.L. Puri and D.A. Ralescu, Limit theorems for random compact sets in Banach spaces, Math. Proc. Cam. Phil. Soc. 97 (1985), 151-158. MR 86c:60024

[12] M.L. Puri and D.A. Ralescu, Fuzzy random variables, J. Math. Anal. Appl. 114 (1986), 409-422. MR 87f:03159

[13] A.W. van der Vaart and J.A. Wellner, Weak convergence and empirical processes, Springer, 1996. MR 97g:60035

[14] W. Weil, An application of the central limit theorem for Banach space valued random variables to the theory of random sets, Z. Wahrscheinlichkeitstheor. Verw. Geb. 60 (1982), 203208. MR 83h:60010

[15] L.A. Zadeh, Fuzzy Sets, Information and Control 8 (1965), 338-353. MR 36:2509

Abt. Math. III, Universität Ulm, 89069 Ulm, Germany

E-mail address: frproske@metronet.de

Current address: Department of Mathematics, University of Oslo, 1053 Blindern, 0316 Oslo, Norway

E-mail address: proske@math.uio.no

Department of Mathematics, Indiana University, Bloomington, Indiana 47405

E-mail address: puri@indiana.edu 